\title{
213. Classification of Carbon Stars by Spectroscopic Scanning
}

\author{
By Yoshio Fujita, M.J.A. \\ Department of Astronomy, University of Tokyo
}

(Comm. Dec. 12, 1967)

In our previous paper (Fujita, 1967) it was suggested that a role of $(6,1)$ and $(7,1)$ bands of $\mathrm{CN}$ red system $\left(\mathrm{A}^{2} \mathrm{II}-X^{2} \Sigma\right)$ on the classification of carbon stars should be of some importance. With some additional observational data obtained by spectroscopic scanning using the same instrument described in our previous paper, this problem will be re-considered in this note.

As some spectral features characterizing carbon stars are remarkable even if the spectral range investigated is rather limited, this method of spectroscopic scanning may be reasonably favourable for the purpose of spectral classification of these stars.

Generally speaking, in the spectral range considered following six features seem to represent most remarkable characteristics of carbon stars from the view point of spectral classification:

$4737 \mathrm{C}_{2}(1,0), 5165 \mathrm{C}_{2}(0,0), 5239 \mathrm{CN}(7,1), 5635 \mathrm{C}_{2}(0,1), 5730 \mathrm{CN}(6,1)$, and $5890-96 \mathrm{Na}$.

As the symbols of criterions which represent the appearance of these features, $\bigcirc$ indicates that the feature of the absorption band or the line considered is clearly recognized, $\times$ indicates that the feature of the absorption band or the line considered disappears or is very weak, and indicates that the band sequences considered partly disappear or are partly very weak.

Seventy-two carbon stars have been investigated according to the various combination of these three criterions which are arranged in order of $\mathrm{Na}, \mathrm{C}_{2}$, and $\mathrm{CN}$. Two typical examples of tracing by spectral scanning are shown in Figs. 1 and 2. After some careful trial, it was clarified that these carbon stars should be reasonably classified into eight types of spectral appearance. The results of assignments of seventy-two carbon stars observed to these types are summarized in Table I. The tentative assignment by the Cclassification is indicated by bracket. It should be mentioned that additional observational data necessitated to revise the assignment of some stars which had been tried in our previous paper and the suffix showing the intensity of $\mathrm{C}_{2}$ band was omitted, because the estimation of $\mathrm{C}_{2}$ intensity from the spectroscopic scanning is sometimes very difficult. 


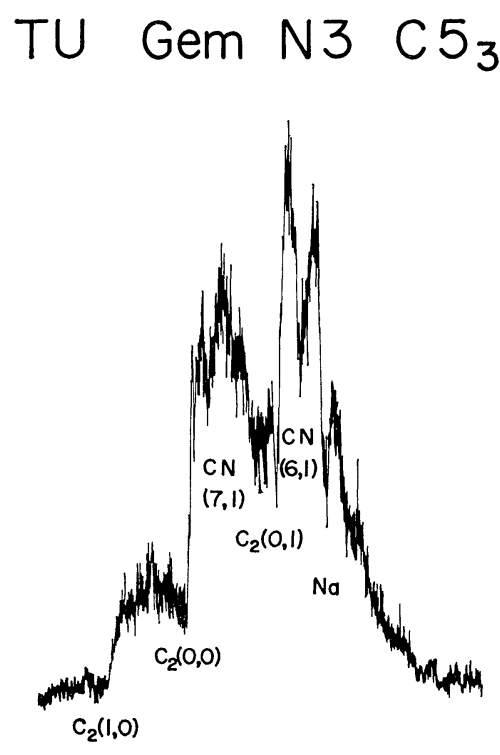

$\begin{array}{lllllllll}11 & 12 & 13 & 14 & 15 & 16 & 17 & 18 & 19\end{array}$

Fig. 1. Typical example of tracing by spectral scanning: TU Gem.
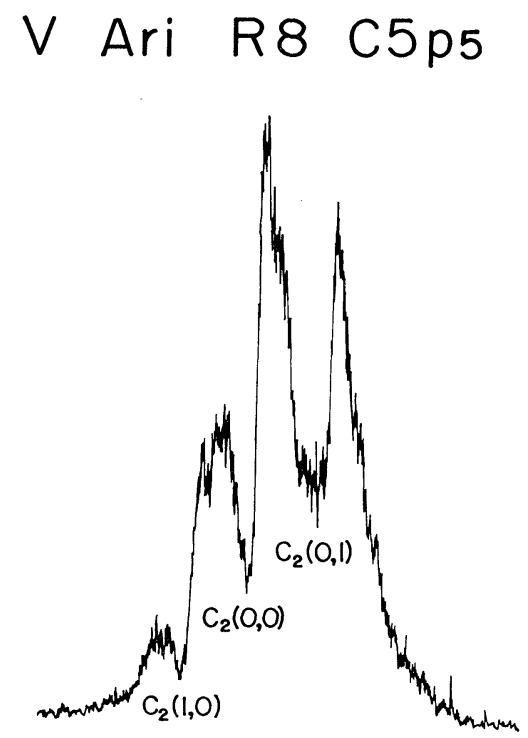

\begin{tabular}{llllll}
10 & 12 & 14 & 16 & 18 & 20 \\
\hline
\end{tabular}

Fig. 2. Typical example of tracing by spectral scanning: V Ari.

The graphical representation showing the correlation between the C-classification and eight types of spectral appearance is shown in Fig. 3. Fig. 4 shows the similar representation by changing the abscissa from the C-classification to the R-N classification. In Fig. 4 the spectral types assigned to $\mathrm{Na}, \mathrm{Nb}$, and $\mathrm{Nc}$ are excluded.

Comparing these two figures, it should be mentioned that the C-classification shows rather better correlation than the R-N classification with the criterion represented by eight types of spectral appearance. The appearance of $\mathrm{CN}$ red system seems to be more sensitive to the C-classification than that of $\mathrm{C}_{2}$ band. The physical explanation of the comparatively good correlation which is shown in Fig. 3 seems difficult. Why the variation of the relative intensity of $\mathrm{C}_{2}$ sequences $(1,0),(0,0)$, and $(0,1)$ and of $\mathrm{CN}$ sequences $(7,1)$, and $(6,1)$ may respectively occur among carbon stars? As there are some stars the spectral features of which are very difficult to make clear distinction among these eight types, it should be understood that such a graphical representation as Fig. 3 is ambiguous and it is possible to find some intermediate types which might be more suitable for representing real situation. The scattering of plots in Fig. 3 will explain such a circumstance. Furthermore, if some 
Table I

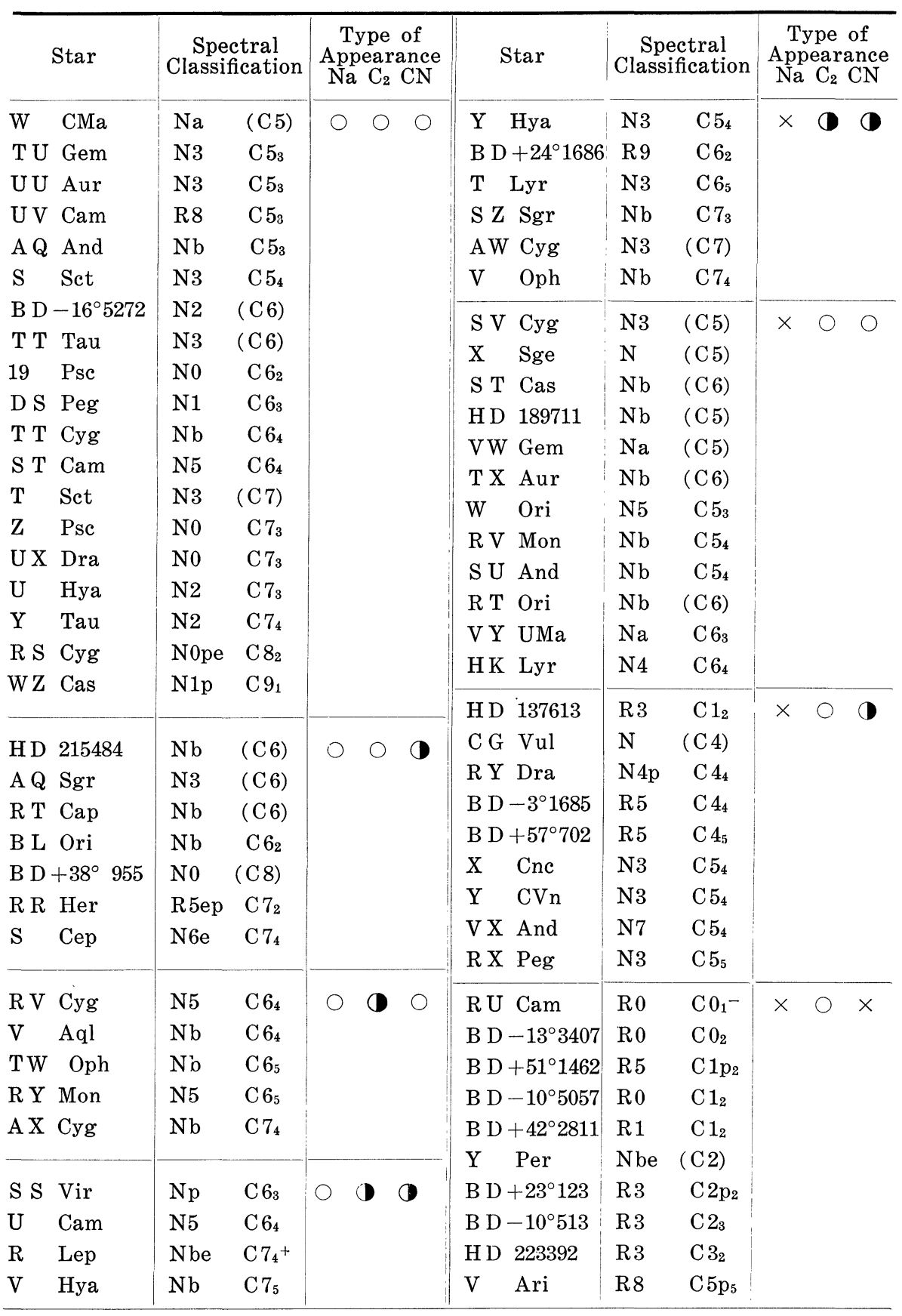




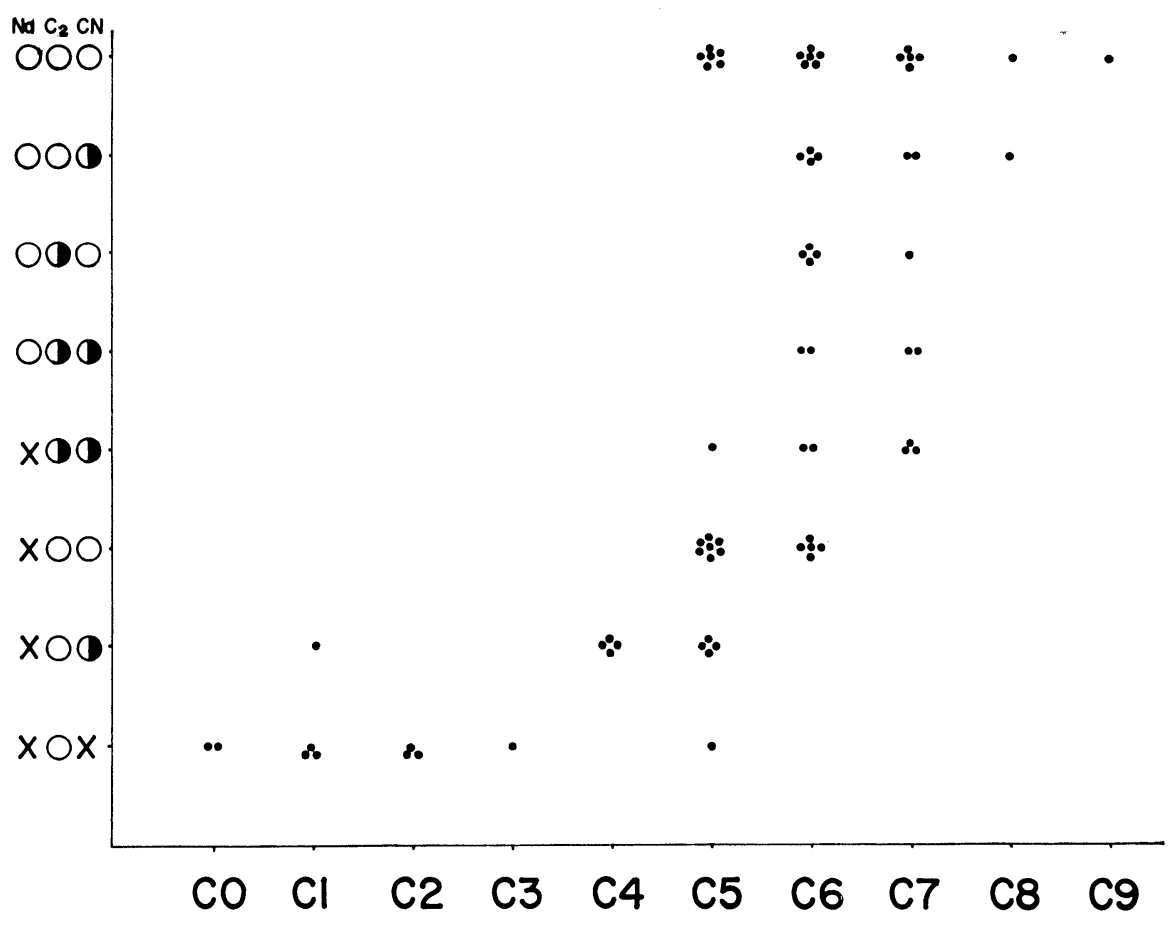

Fig. 3. Correlation between the C-classification and eight types of spectral appearance in the spectroscopic scanning investigated.

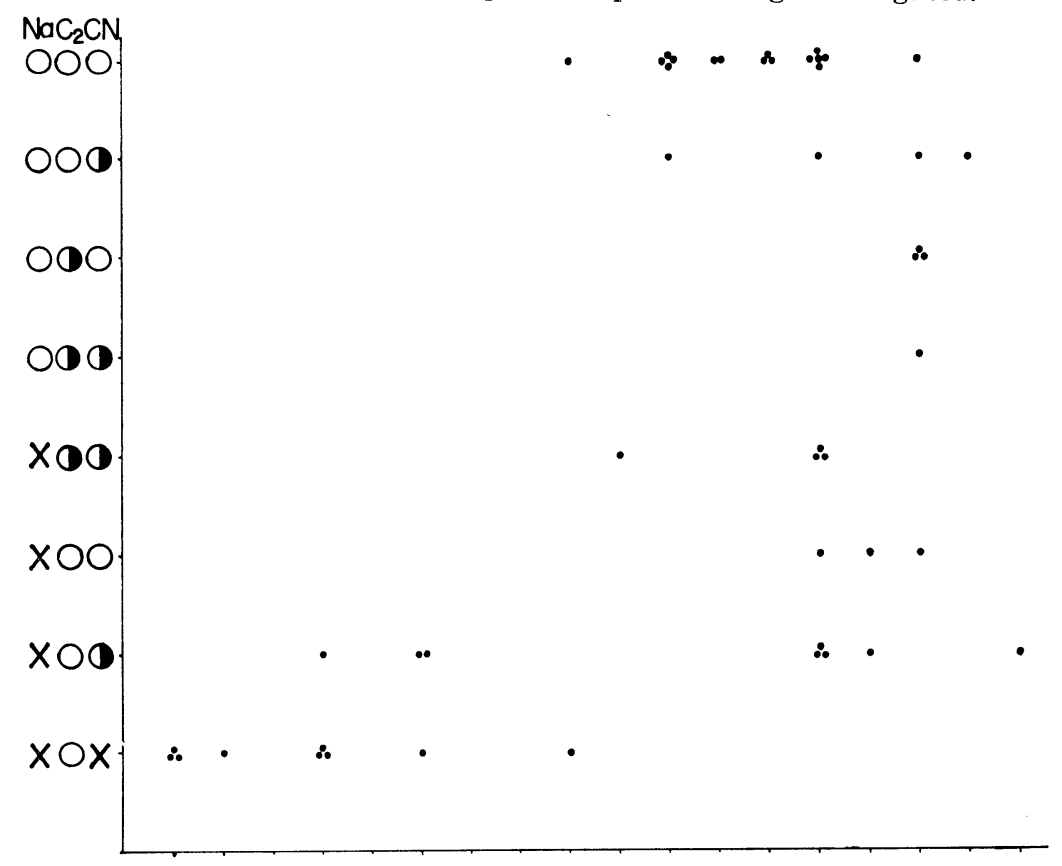

RO RI R2 R3 R4 R5 R6 R7 R8 R9 NONI N2 N3 N4 N5 N6N7

Fig. 4. Correlation between the $R-N$ classification and eight types of spectral appearance in the spectroscopic scanning investigated. 
quantitative consideration could be added in connection with the relative intensity of $\mathrm{C}_{2}$ and $\mathrm{CN}$ band sequences, it might help our idea of spectral classification above described.

In conclusion, I wish to express my special thanks to members of the staff of the Okayama Astrophysical Observatory for their assistance in observations. My thanks are also due to Miss M. Matsugaki for her assistance in preparing this manuscript.

\section{Reference}

Fujita, Y. (1967): Proc. Japan Acad., 43, 472. 